\title{
Stability of a mine excavator with a variable cab position
}

\author{
Vladimir Velikanov ${ }^{1, *}$, Natalja Dyorina ${ }^{1}$, and Olga Panfilova ${ }^{1}$ \\ ${ }^{1}$ Nosov Magnitogorsk State Technical University, Magnitogorsk, Russia
}

\begin{abstract}
The publication deals with the problem of assessing the mining excavator stability with a variable cab position. It is determined that the efficiency of the excavator cycle operations largely depends on the position of the operator with respect to the boom and body, the glazing area of the cabin, the dimensions of the windows, the width and the number of jumpers. A technical solution is proposed to increase the visibility of mining excavators' cabs. The design of the mechanism makes it possible to advance the efficiency of using quarry excavators by changing the position of the cabin both in the vertical plane and in the horizontal one. Counterweight mass, an assessment of the turntable equilibrium and the stability of a mining excavator with a variable cab position are performed using standard «MS Excel» tools.
\end{abstract}

\section{Introduction}

The improvement of the methods determining visibility, illumination of working spaces and arranging the operator's workplace as components of the excavator's controllability is determined by the need to support the competitiveness of domestic excavator models.

Visibility is considered to be one of the main ergonomic indicators of the mining excavator manageability, which influences the information support and the operator's preparation for making decisions. In general, the visibility is understood as a constructive property that characterizes the objective possibility and conditions for the operator to perceive visual information necessary for the safe and efficient machine control $[1,2]$.

Concerning the quarry excavator, we will assume that the quality of visibility and visibility of the given observation objects from the mining excavator operator's workplace depends on the cabin position with respect to the boom and body, the glazing area, the dimensions of the glass, the width and the number of lintels [1-4].

There is a wider body of research $[5,6]$ concerning the indicators of constructive excellence determining the working conditions for the excavator operator. They outlined the importance of the visual information content of the production area and the operator's working space. On the basis of orthogonal drawings of the excavator and full-scale models of the glance from the operator's workplace, the obligatory perception zones are defined, and the visibility values ranges of the primary and periodic observation objects are given. The

\footnotetext{
*Corresponding author: rizhik_00@mail.ru
} 
methodical basis of the research was the basic methodology "Constructing visibility pictures from the excavator operator's workplace", based on GOST 12.2.130-91.

As a review measure for each of the given observation objects, a $K_{i O}$ viewing factor ranging from 0 to 1 is set. The survey object overview contains a surface, a line or an angle. The coefficient of the surface or line survey is defined as the ratio of the area or line of the visible object part to its total area or line of the required survey. For point objects, the view coefficient is equal to one if the objects are in the field of view, and to zero if they are out of sight [5].

Observation objects on mining excavators can be divided into two groups:

- primary observation objects - slaughter, a bucket with a handle, transport, a boom;

- periodic observation objects - the control panel, caterpillars, the face top edge.

The technical solution for raising and lowering the excavator cab is used by Caterpillar (models MH3049, MH3059 and 330D MH), Volvo (model EC480D with Step Safe Cab function), and also in reloaders made on the wheeled hydraulic excavators.

The works $[1,2]$ proposed the technical solution implementation to improve the visibility of the mining excavators' cabins. The mechanism design makes it possible to increase the efficiency of using quarry excavators by changing the cabin position both in the vertical plane and in the horizontal one. As a basis, the use of a lever-type lift is accepted. In the specialized program Autodesk Inventor, a parametric 3D mechanism prototype is designed and calculated by the finite-element method in the basic design positions: the initial moment of lifting, when lifting to the maximum height, at the maximum outreach.

The following loads, acting on the mechanism design, are simulated: from the own design mass; concentrated force from the cabin weight, and also it is possible to vary the cabin mass and the speed of its lifting.

The mechanism consists of a support base on which two parallel scissor mechanisms are mounted, consisting of crisscross levers, a lifting platform on which a modular cab and hydraulic cylinders are installed. Controlling the cab movement is carried out with the help of a remote control panel mounted in it.

In the construction of a career excavator the use of the mechanism for changing the cab position both in the vertical plane and in the horizontal one determines the need for the machine stability assessment.

\section{Methodology}

Equilibrium determination of the swivel platform and the excavator stability is the goal of the static calculation of single-bucket quarry excavators. The balancing of the turntable is achieved by appropriately placing all the mechanisms on it and choosing the counterweight mass $[7,8]$.

For a straight shovel, the counterweight mass is determined for the two calculated positions (Fig. 1) [9-12].

1. The ladle is lowered on the supporting surface (the bucket and the handle weight do not create a moment).

2. The ladle is pushed out on $2 / 3$ of a handle start.

At the first design position, as a rule, the excavator stability is ensured. As for the second position, the counterweight mass is calculated from the moment equation for point $\mathrm{B}$ (Figure 1) using the formula:

$$
m_{c}^{I I}=\frac{G_{b}\left(r_{b}-R_{o}\right)+G_{h} r_{h}+G_{l+r} r_{l}-G_{p l}\left(r_{p l}+R_{o}\right)}{g\left(r_{c}+R_{o}\right)} .
$$


The turning part equilibrium of the mining excavators of the Uralmashzavod PJSC is determined from the condition for the failure of the resultant vertical forces at the loaded ladle, which is at the maximum reach, beyond the roller circle with a margin of $8-10 \%$, precisely under these conditions the weighed counterweight mass is determined.

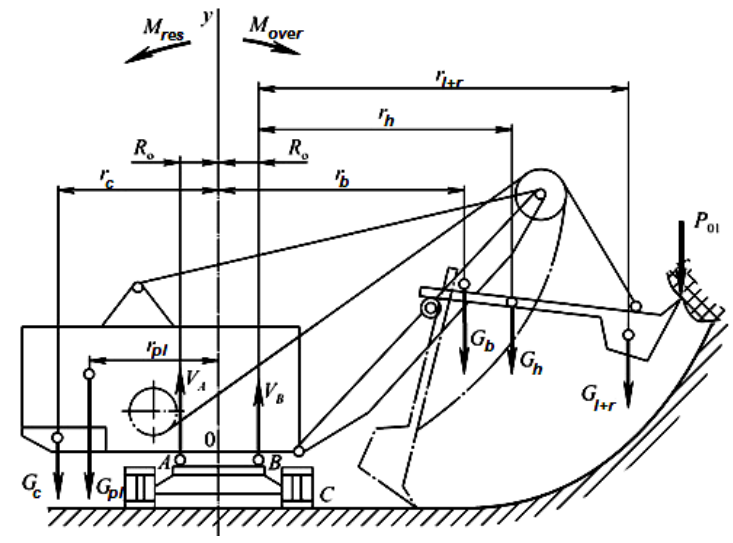

Fig. 1. Equilibrium determination scheme for a straight shovel.

The mining excavator stability characterizes the machine ability to withstand external loads, including the gravity forces of the rock in the bucket, as well as the components of the excavator, the resistance of the digging and inertia forces, which do not allow to overturn and move the support part of the mining excavator relative to the soil base $[7,8]$.

The stability of a mining excavator is characterized by a stability coefficient:

$$
k_{s}=\frac{\text { Mres }}{\text { Mover }},
$$

where Mres - moment with respect to the tilting edge of the restoring (restraining) forces $\mathrm{Nm}$; Mover - the moment relative to the tripping edge of overturning forces $\mathrm{Nm}$.

In modern methods of assessing the excavators stability, there are significant differences in the approaches to taking into account the types of forces that affect the values of the holding and tripping moments.

The methodology of the Rostechnadzor Rules provides for the machine stability calculation, both in the working and non-operating state. The stability factor is calculated in two ways. The first way takes into account the actions of additional loads, for example, wind, inertial forces, wheras in the second one additional loads and inclination are not taken into account.

The technique of the Kovrov Excavator Plant determines the coefficient of static stability, taking into account only the gravity forces of the excavator construction components and rock in the ladle.

The All-Union Research and Developmnt Establishment stroidormash method takes into account the effect of centrifugal forces acting on the the excavator design components when the platform is turned.

According to the Karasev-Zhiweinov technique, the verification of the stability conditions includes all possible positions of the excavator and working equipment, to take into account the combination of loads that are dangerous from the point of view of the stability loss. The stability factor is determined taking into account gravity forces, centrifugal forces, inertia forces, including Coriolis forces, as well as wind forces acting on the structural elements when the platform and the working equipment are mutually rotated [11]. 
The stability of a mining excavator equipped with a straight shovel is usually determined for four cases [11]:

1. When the obstacle is separated, for example in the form of oversized, the excavator is installed in a horizontal plane; the working equipment is installed across the running gear; the ladle is empty; the bucket teeth are located at the level of the pressure shaft; the boom is inclined to the horizon at an angle of $45^{\circ}$; lifting ropes are vertical; the maximum force is realized on the cutting edge of the bucket.

2. In the standard operation of the excavator it is assumed that the next machine is installed in a horizontal plane, the working equipment is located across the running device. The handle is horizontal and maximally extended, the boom is inclined to the horizon at an angle of $45^{\circ}$, the bucket is filled with a rock mass, the operating force acts in the lifting ropes, and the pressure force is equal to half the working one, which moves the bucket towards itself, directed to the face. Tilting and holding moments are calculated by the formula:

$$
k_{s}=\frac{\text { Mres }}{\text { Mover }}=\frac{G_{t r} r_{t r}+G_{c} r_{c}+G_{\mathrm{pl}} r_{p l}}{P_{01} l_{3}+G_{l+r} l_{l}+G_{h} l_{h}+G_{b} l_{b}},
$$

where $G_{\mathrm{tr}}, G_{\mathrm{c}}, G_{\mathrm{pl}}, G_{l+r}, G_{\mathrm{h}}, G_{\mathrm{b}}$ the weight, respectively the trolley, the counterweight, the swing platform, the handle, the boom, the ladle with the rock $\mathrm{kN} ; P_{01}$ is calculated strength of rock resistance, $\mathrm{kN} ; r_{t r}, r_{\mathrm{c}}, r_{\mathrm{pl}}, l_{3}, l_{l}, l_{h}, l_{b}$ are the shoulders of the action of forces, $\mathrm{m}$.

$$
P_{01}=k_{f} \cdot E \cdot 10^{3}\left(H_{\mathrm{b}} \cdot k_{\mathrm{p}}\right)^{-1},
$$

where $k_{f}$ is the coefficient of rocks resistance, $\mathrm{MPa} ; E$ is excavator ladle capacity $\mathrm{m}^{3} ; H_{\mathrm{b}}$ is height of the pressure shaft, $\mathrm{m} ; k_{\mathrm{p}}$ is the coefficient of rocks loosening.

The excavator moves uphill in the transport position. The excavator boom with cable-block control is inclined to the bearing surface at the smallest angle (about $30^{\circ}$ ), the bucket is at the ground, and the handle is fully extended.

3. The excavator moves in a transport position under the slope, the handle hangs vertically, the boom of the excavator with cable-block control is inclined to the supporting surface at the maximum angle.

The overall stability of the quarry excavator is checked from the condition of nonoccurrence of the resultant vertical forces, including forces on the bucket teeth during digging, the weight of the running trolley and the weight of the embedded counterweight, calculated for the turning part equilibrium, beyond the supporting contour of the running trolley. As a rule, the stability factor is not less than 1.4, which is quite enough.

\section{Results}

Counterweight mass calculation, turntable equilibrium assessment and the stability of a mining excavator with a variable cab position is performed according to a known technique presented in the reference literature [7, 8], using standard "MS Excel" tools. The initial data, namely the nodes masses of the mechanical system of CME excavators (Table 1), as well as the location of the mass centres of the main elements relative to the tip of the overturn (for example, the CME -10 excavator) are indicated in Table $2[15,16]$. If necessary, the linear dimensions of the $L_{i}(\mathrm{~m})$, structural elements can be determined depending on the mass of the excavator $m_{e}(\mathrm{t})$, and the proportionality coefficient $k_{i}$ (taken from the reference literature) according to the empirical expression: $L_{i}=k_{i} \sqrt[3]{m_{e}}$.

Example: calculation $k_{\mathrm{s}}$ for CME-10 relative to the tripping edge (point C) (Fig. 2). 


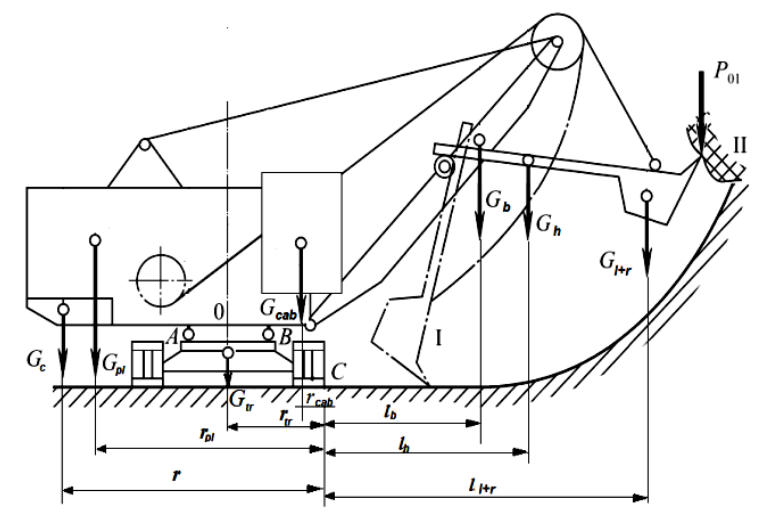

Fig. 2. Scheme for calculating the mining excavator stability with a variable cab position

The moment with respect to the tripping edge of the holding forces

$$
\begin{gathered}
\text { Mres }=G_{t r} r_{t r}+G_{c} r_{c}+G_{p l} r_{p l}+G_{c a b} r_{c a b} \\
=1050 \cdot 3,5+500 \cdot 10,3+1540 \cdot 5,5+5,3 \cdot 0,56=17297,96 \mathrm{kNm} .
\end{gathered}
$$

The moment relative to the overturning forces tip:

$$
\begin{gathered}
\text { Mover }=P_{01} l_{3}+G_{l+r} l_{l}+G_{h} l_{h}+G_{b} l_{b} \\
=338 \cdot 12,6+410 \cdot 12,2+115 \cdot 9,2+274 \cdot 7,7=12220,7 \mathrm{kNm} . \\
k_{s}=\frac{\text { Mres }}{\text { Mover }}=\frac{17297,96}{12220,7}=1,41>1 .
\end{gathered}
$$

Table 1. Nodes masses of the mechanical system for some types of mining excavators

\begin{tabular}{|l|c|c|c|c|c|}
\hline $\begin{array}{c}\text { № } \\
\text { Part } \\
\text { number }\end{array}$ & Node & CME-5A & CME -10 & CME -12,5 & CME -20A \\
\hline 1. & Constructive mass without counterweight, $\mathrm{t}$ & 154 & 350 & 638 & 900 \\
\hline 2. & Counterweight, $\mathrm{t}$ & 40 & 50 & 20 & 160 \\
\hline 3. & Swing platform, $\mathrm{t}$ & 58,3 & 154 & 287 & 405 \\
\hline 4. & Boom assembly, $\mathrm{t}$ & 17,3 & 27,4 & 62,1 & 65 \\
\hline 5. & Handle, $\mathrm{t}$ & 7,75 & 11,5 & 18,3 & 24,7 \\
\hline 6. & Ladle, $\mathrm{t}$ & 9,94 & 16,2 & 27 & 35 \\
\hline 7. & Lower and crawler turntable, $\mathrm{t}$ & 41,4 & 105 & 191 & 320 \\
\hline 8. & Operator cab, $\mathrm{t}$ & $1,1-2,6$ & 5,3 & 7,5 & 5,3 \\
\hline 9. & Maximum mass of rock in the ladle, $\mathrm{t}$ & 9 & 25 & 60 & 96 \\
\hline
\end{tabular}


Table 2. The centers coordinates of the nodes mass of the excavator CME-10 relative to the tripping edge

\begin{tabular}{|c|c|c|c|c|c|}
\hline $\begin{array}{c}\text { № } \\
\text { Part } \\
\text { number }\end{array}$ & Element & $\begin{array}{c}\text { Identify } \\
\text { cation }\end{array}$ & $\begin{array}{c}\text { Value, } \\
\mathrm{m}\end{array}$ & $\begin{array}{c}\text { Identify } \\
\text { cation }\end{array}$ & $\begin{array}{c}\text { Value, } \\
\mathrm{m}\end{array}$ \\
\hline 1. & Counterweight & $X_{\mathrm{co}}\left(\mathrm{r}_{\mathrm{co}}\right)$ & $-10,3$ & $Y_{\text {co }}$ & 0,53 \\
\hline 2. & Swing platform & $X_{\mathrm{t}}\left(\mathrm{r}_{\mathrm{t}}\right)$ & $-5,5$ & $Y_{\text {tur }}$ & 3,13 \\
\hline 3. & Boom suspension, struts and ropes & $X_{\text {sus }}$ & 3,9 & $Y_{\text {sus }}$ & 9,69 \\
\hline 4. & Boom assembly & $X_{\text {bo }}\left(l_{a}\right)$ & 7,7 & $Y_{\text {bo }}$ & 6,62 \\
\hline 5. & Stairs and platforms & $X_{\text {st }}$ & 8,4 & $Y_{\text {st }}$ & 7,7 \\
\hline 6. & Handle & $X_{\text {han } \max }\left(l_{p}\right)$ & 9,2 & $Y_{\text {han } \max }$ & - \\
\hline 7. & Lower and crawler turntable & $X_{\mathrm{T}}\left(\mathrm{r}_{\mathrm{T}}\right)$ & $-3,5$ & $Y_{\mathrm{T}}$ & - \\
\hline 8. & Operator cab & $X_{\text {cab }}\left(\mathrm{r}_{\mathrm{cab}}\right)$ & $-0,56$ & $Y_{\text {cab }}$ & - \\
\hline 9. & Ladle + rock & $X_{\text {gr } \max }\left(l_{\kappa+n}\right)$ & 12,2 & $Y_{\text {gr } \max }$ & - \\
\hline 10. & Thrust axis & $X_{\text {t.a }}$ & 8,14 & $Y_{\text {t.a }}$ & 7,7 \\
\hline
\end{tabular}

The use of standard «MS Excel» tools allowed obtaining the following results for some samples of mining excavators, table 3.

Table 3. Estimated values of the safety factor

\begin{tabular}{|c|c|c|c|c|}
\hline & CME-5A & CME - 10 & CME -12,5 & CME -20A \\
\hline$k_{s}$ & 1,38 & 1,41 & 1,43 & 1,42 \\
\hline
\end{tabular}

\section{Conclusion}

Thus, the study of the mine excavator stability with a variable cab position made it possible to prove the full validity of the taken technical decision, since the calculated design values of the stability factor correspond to the requirements (stability coefficient should be at least 1.4, Uralmashzavod PJSC). The developed technical solution for the modernization of mine excavators is oriented to practical application and extends the technological capabilities of excavators by using a cabin lift. The introduction of the device will allow increasing the efficiency of performing technological tasks by using the operational capabilities of the machine more fully.

\section{References}

1. V.S. Velikanov, A.V. Kozyr', I.G. Usov, I.A. Grishin, K.V. Burmistrov, Mining industry. 6 (2016)

2. V.S. Velikanov, A.V Kozyr, N.V. Dyorina, Procedia Engineering. 206(2017)

3. V.S. Velikanov, Mining Journal. 9 (2012)

4. V.S. Velikanov, E.A. Ilina, N.V. Dyorina, Procedia Engineering. 150 (2016)

5. N.N. Gruzdeva, L.P. Khomyakova, V.G. Khusainov, Mining industry. 3 (2002)

6. Ye.A. Volkov, Research of efficiency of working processes of excavator equipment using remote control (Belgorod, 2016) 
7. R.YU. Poderni, The mechanical equipment of open-cast mines: the textbook for high schools (Izd-vo MGGU, Moscow, 2007)

8. M.I. Shchadova, R.Yu. Poderni, Handbook of mechanics of open works. Excavation transport machines of cyclic action (Nedra, Moscow, 1989)

9. N.N. Zhiveynov, G.N. Karasev, Bulletin of Machine Building. 7 (1997)

10. Ye. S. Buryak, Mountain machines and equipment: Methodological instructions for practical exercises (IPC SAFU, Arkhangelsk, 2015)

11. A.A. Kholyavko, P.A. Kapralov, A.A. Buyeva, Youth and Science. (2014)

12. A.V. Semenov, S.I. Vakhrushev, Master's Journal. 2 (2016)

13. V.S. Velikanov, I.G. Usov, A.A. Abdrakhmanov, I.I. Usov, Mining Journal. 12 (2017)

14. V.S. Velikanov, N.V. Dyorina, A.A. Abdrakhmanov, Matec web of conferences, 129 (2017)

15. Uralmashzavod, Brochures with technical characteristics [Electronic resource], https://www.uralmash.ru/production/karernye_gusenichnye_ekskavatory_

mekhanicheskie_lopaty /.

16. Brochures with technical characteristics [Electronic resource], - http://izkartex.com/karernyie-ekskavatory. 